EOMmun Communication et organisation

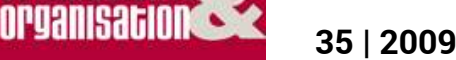

Repenser la communication dans les organisations publiques

\title{
Les organisations publiques : des institutions
} communicantes?

Le cas des Caisses d'Allocations Familiales

Jacques Bonnet, Rosette Bonnet et Sabrina Hélaine-Pinsard

\section{OpenEdition \\ Journals}

Édition électronique

URL : http://journals.openedition.org/communicationorganisation/782

DOI : 10.4000/communicationorganisation.782

ISSN : 1775-3546

Éditeur

Presses universitaires de Bordeaux

Édition imprimée

Date de publication : 1 juin 2009

Pagination : 136-147

ISSN : $1168-5549$

Référence électronique

Jacques Bonnet, Rosette Bonnet et Sabrina Hélaine-Pinsard, « Les organisations publiques : des

institutions communicantes? », Communication et organisation [En ligne], 35 | 2009, mis en ligne le 01 décembre 2012, consulté le 30 avril 2019. URL : http://journals.openedition.org/

communicationorganisation/782 ; DOI : 10.4000/communicationorganisation.782 
Dossier : Repenser la communication des organisations publiques

\title{
Résumé
}

L'objectif de cet article est de proposer une approche compréhensive de la communication des organisations publiques à travers le cas des Caisses d'Allocations Familiales (CAF). Les travaux dont il est issu ont eu pour objet d'interroger les représentations des cadres, chargés de communication et directeurs des CAF autour de la notion d'institution communicante. Il s'agit notamment de préciser si celle-ci relève d'un artefact ou pourrait -et à quelles conditions- constituer un modèle valide pour analyser la communication des organisations publiques.

Mots-clés

Institution communicante, organisations publiques, caisses d'allocations familiales, approche communicationnelle, représentations des cadres.

\begin{abstract}
The aim of this article is to propose a comprehensive approach to the concept of communication within State Department using the example of the Family Allowance Department. The objective of the research carried out was to question how the executives, communication analysts and directors of the Family Allowance Department represented in their minds the notion of an institution which communicates effectively. It is necessary to determine whether this notion is merely an artefact or whether it could, and if so under circumstances, constitute a valid model that might be used to analyse the communication systems within other State Department.
\end{abstract}

\section{Key-Words}

Communicative institution, State Departments, Family Allowance Department, communicative approach, executives representations.

\section{Auteurs}

Jacques Bonnet est Professeur en Sciences de l'information et de la communication à Agrosup Dijon. Il dirige l'équipe de recherche Cimeos/Cosmos (EA 4177) à l'Université de Bourgogne.

Rosette Bonnet est Maître de Conférences HDR en Sciences de l'information et de la communication à Agrosup Dijon. Elle est membre de l'équipe de recherche Cimeos/Cosmos à l'Université de Bourgogne.

Sabrina Hélaine-Pinsard est Chargée de communication à la Caisse d'Allocations Familiales du Sud Finistère. 


\title{
Les organisations publiques : des institutions communicantes? Le cas des Caisses d'Allocations Familiales
}

\author{
Jacques Bonnet, Rosette Bonnet, Sabrina Hélaine-Pinsard
}

\author{
jacques.bonnet.couchey@orange.fr \\ rosette.bonnet@orange.fr \\ sabrina.helaine@wanadoo.fr
}

Institution en charge de l'accompagnement des familles et des personnes dans le domaine de la parentalité, du logement et de la lutte contre la précarité, les Allocations familiales sont aujourd'hui interpellées pour répondre aux nouvelles attentes sociales des publics et à des exigences accrues de qualité de service et de réduction de coûts. Malgré une modernisation avérée, une représentation d'administration lourde et complexe subsiste à leur sujet auprès d'une partie du grand public, image dont les médias se font régulièrement l'écho.

Dans le même temps, parce qu'elles incarnent, en termes de services, les attentes fortes des usagers au regard de la sphère démocratique et sociale (Lipovetsky, 2006 ; D'Iribarne, 2006) les Caisses d'allocations familiales (CAF) apparaissent comme un ultime rempart face aux fragilisations sociales qui caractérisent la société hypermoderne. Dans ce contexte, la Branche Famille et les CAF affichent des intentions en matière de communication : moderniser leur image, soutenir leur performance, améliorer la qualité des relations avec les usagers et les partenaires, faire évoluer les pratiques de travail, susciter l'engagement des personnels en interne... Ces enjeux font d'autant plus débat que l'adaptation aux attentes sociales et économiques est fréquemment posée par les pouvoirs publics comme la condition de la pérennité des organismes concernés. Les CAF se trouvent donc interpellées quant à leur capacité à s'afficher et à intervenir en tant «qu'institutions communicantes». Si la notion d'«organisation apprenante » a largement été répandue, et a fait l'objet de nombreux travaux (Mallet, 1996; Ardoino, 1996), le concept d'institution communicante, plus récent et auquel se réfèrent, sous plusieurs aspects, les Conventions d'objectifs et de gestion passées entre la Branche Famille de la Sécurité sociale et l'État, appelle de nouvelles questions quant à sa pertinence et à sa capacité à témoigner des pratiques sociales liées au travail au sein des organismes. 
Afin de situer ce concept en émergence, nous dirons tout d'abord qu'il a été plus fréquemment utilisé pour souligner l'importance des interactions entre une organisation et ses environnements (Boneu, 1990 ; Orgogozo, 1998) et peu à propos des services publics. Dès lors, « institution » et « communicante » sont-ils deux termes compatibles, l'un se référant à une perspective de nature structuraliste, voire déterministe, de l'organisation du travail, l'autre mettant en débat les interactions dans et autour des CAF?

Nous posons ici l'hypothèse selon laquelle la notion «d'institution communicante » renvoie, au-delà des questions de structure, mais en contrepoint de ces dernières, à l'appréhension des interinfluences qui se jouent aux plans sensible et symbolique (Bougnoux, 1993) au sein des CAF et dans leurs relations avec leurs environnements, leurs partenaires et leurs usagers. De ce fait, nous questionnons ici l'institution communicante en termes de creuset éventuel et potentiel pour des interactions fondées sur une construction négociée du sens de l'action collective ainsi que sur un espace et des processus de médiation des significations (Lamizet, 1995) liées aux activités propres à ces organismes.

Après avoir précisé l'approche conceptuelle et méthodologique que nous avons retenue, nous consacrerons une part essentielle de cet article à fournir les résultats d'une recherche à vocation exploratoire menée auprès des personnels d'encadrement de plusieurs CAF. Nous chercherons ainsi à identifier les écarts et les convergences entre d'une part, le recours de ces organismes à l'affichage de l'importance de la communication pour leur fonctionnement interne et pour la qualité de service visée envers l'usager et, d'autre part, les représentations et les postures des cadres chargés de relayer cette intention communicationnelle auprès des personnels et des services. Sur la base de ces résultats, nous tenterons d'esquisser une modélisation des processus communicationnels témoignant d'une pertinence du concept d'institution communicante dans les organisations publiques. Enfin, et toujours à la lumière du sens donné à la communication par les cadres, nous mettrons ce modèle en perspective au regard du contexte managérial et organisationnel des CAF.

\section{Approche conceptuelle et méthodologique}

Nous interrogeons ici l'institution communicante en termes de système de significations construit et éventuellement porté et partagé par ses personnels, dont ses cadres. De ce point de vue et à la lumière des travaux d'E. Morin (1990) et de J.-L. Le Moigne (1999) à propos de la complexité organisationnelle et notamment du concept d'auto- 
organisation, nous nous référons à une approche compréhensive de l'émergence de la fonction Communication au sein des CAF ainsi que des représentations qui lui sont associées chez les cadres.

Nous postulons donc que ces représentations portant, par exemple, sur la validité, la pertinence et la plus value d'une intention et d'activités de nature communicationnelle de la part et au sein des CAF, relèvent d'une élaboration individuelle et collective traduisant une interprétation de la réalité et fournissant des référents pour les discours et les actes de travail. Notre attention s'est donc portée sur la genèse des processus de signification (Eco, 1997 ; Lamizet, 2006) au sein des groupes sociaux et des communautés de travail que constituent les $\mathrm{CAF}$ et leurs services, et tout particulièrement leurs cadres, en raison de leur position d'interface fonctionnelle et symbolique. Nous considérons donc ces cadres comme des traducteurs, des producteurs et des acteurs de sens et non comme des récepteurs passifs au regard de l'intention communicationnelle affichée institutionnellement. Le concept de sens auquel nous nous référons questionne ainsi les représentations des cadres relatives aux orientations, aux intentions et à la plus-value potentielle (Bougnoux, 2002 ; Weil-Barais, 2005) de la fonction et des activités de communication au sein des CAF, telles que se les représentent les cadres de ces organismes.

Pour Abric, le concept de représentation se définit comme « produit et processus d'une activité mentale par laquelle un individu ou un groupe reconstitue le réel auquel il est confronté et lui attribue une signification ». Cette acception gagne, selon nous, à être combinée avec celle développée par D. Jodelet (2007) pour qui les représentations sociales se forgent et s'expriment en termes de concepts, d'évocations mentales et de représentations liées à l'action. En cela, c'est à partir d'une approche sociocognitive des significations attribuées par les cadres des CAF à la prescription institutionnelle et aux activités professionnelles relatives à la communication (Bonnet, Bonnet, 2003), que cette recherche vise à caractériser, de façon originale, les contours de la notion d'institution communicante.

Pour ce faire, dix-huit entretiens semi-directifs ont été menés dans huit organismes entre janvier et mai 2007 auprès de cadres, directeurs et chargés de communication de la Caisse nationale des allocations familiales et de CAF. Pour chacun des thèmes abordés (rapports entre organisation et stratégie, conceptions des cadres au regard de la fonction communication, place, rôles et effets de la communication dans les pratiques managériales, etc.), les données collectées ont été 
Dossier : Repenser la communication des organisations publiques

codées et analysées en reprenant les critères liés aux concepts énoncés ci-dessus.

La notion d'institution communicante vue par les cadres des CAF

Nous présentons ci-dessous les principaux résultats issus de l'analyse des entretiens semi-directifs.

\section{Les représentations des cadres vis-à-vis de la dimension communicationnelle de leurs organismes}

Si les cadres des CAF disent majoritairement reconnaître à leur institution une volonté de communiquer qui s'est accrue ces dernières années, cette démarche volontaire ne suffit pas, selon eux, pour qualifier leur institution de «communicante»: "Jusque dans les années 90, notre institution n'était pas véritablement communicante. Les Conventions d'objectifs et de gestion ont accru le besoin de communiquer. Aujourd'hui, il y a une véritable dynamique de communication, même si à l'évidence, il demeure des problèmes de communication sur les décisions politiques. ${ }^{\mathbf{1 2 5}{ }}$

Ils expriment également un écart entre ce qu'ils perçoivent comme faisant référence à de l'information, et ce qu'ils déclarent attendre : une fonction communication offrant plus d'interactions, facilitant davantage de liens avec le management et l'organisme dans son ensemble. Enfin, leurs évocations traduisent des attentes qui relèvent d'une conception de la communication basée sur les relations interpersonnelles et de groupes, dans un système devenu plus complexe. Il y a donc ici un effet de dissonance ${ }^{\mathbf{1 2 6}}$ entre les attentes exprimées par les cadres en termes d'interactions sociales et une réalité professionnelle vécue essentiellement de manière transmissive.

Dès lors, quelles représentations les cadres des CAF se font-ils d'une « institution » qu'ils pourraient qualifier de «communicante »? Une culture de l'échange, une posture d'ouverture (d'empathie) et le temps pris pour communiquer sont des indicateurs, selon eux, de ce qui construit «une institution communicante ». A contrario, ils relèvent des freins à cette construction en raison d'une part "d'une trop grande frilosité et d'un manque de moyens » et, d'autre part, d'un écart avec les pratiques et les moyens consacrés par d'autres entreprises à la communication : « Notre service public est parmi ceux

\footnotetext{
${ }^{125}$ Les citations, en italique, sont extraites des entretiens semi-directifs.

${ }^{126} \mathrm{Ph}$. Breton (2006) fait le constat d'un problème général de dissonance entre le fort attachement aux valeurs démocratiques dans les sociétés modernes et la faible prévalence des pratiques démocratiques.
} 
qui communiquent le moins... Avec ça, je ne suis même pas capable de dire quelle image de nous peut bien avoir le grand public! ». Les cadres interrogés mentionnent enfin un manque de clarté sur les ambitions et le positionnement du service public des Allocations familiales et questionnent ainsi un déficit de sens au plan institutionnel: "Je ne peux pas dire que les CAF sont des institutions communicantes, car il n'y a pas de visibilité sur les ambitions de la Branche. C'est à nous d'aller les chercher, de les reconstruire. J'ai le sentiment que personne n'est capable de dire clairement notre identité et notre projet. " Cette dernière remarque renvoie à un problème d'identité de l'institution qui se pose en termes de compréhension de ses intentions (quel est son projet ?) et de la plus-value recherchée au travers de la communication (pourquoi l'institution communique-telle, en vue de quelle expression de ses métiers, du rapport à l'usager et du service offert ?).

Valeurs exprimées par les cadres au regard de la notion d'institution communicante

La première valeur évoquée par les cadres des CAF est l'authenticité : "Etre authentique », "sincère », " au clair sur ce qu'il y a à faire passer $\gg$. En exprimant leur attachement à la valeur d'authenticité, ils se situent à la fois dans le prolongement et en rupture avec les valeurs traditionnelles des institutions de service public. S'ils indiquent que la communication de l'institution doit se démarquer des valeurs des entreprises privées qui mettent, selon eux, davantage en avant les valeurs d'efficacité ou de rentabilité, ils ne formulent plus ces valeurs en termes de neutralité, d'objectivité, mais en termes de sincérité, d'authenticité, ce qui renvoie davantage à une relation plus sensible et incarnée $e^{127}$. Le second groupe de valeurs mis en avant par les cadres est lié à «l'interactivité ${ }^{128}$ » et à «la réactivité », qu'ils désignent aussi «impulsion », «dynamisme », ce qui, selon eux, permettrait à l'institution " de communiquer de manière moins confidentielle», «d'être plus adaptable, en un mot d'être plus moderne». Ces valeurs sont affirmées comme conditions et signes de modernité. Si elles sont

\footnotetext{
${ }^{127}$ Nous reprenons ici ce terme en référence à la «position de parole incarnée » décrite par P. Vermersch (1993) et F. Varela (2004), qui désigne le fait que le sujet soit véritablement en relation vivante et authentique avec ce dont il parle au moment où il parle et qui regarde la cognition comme reliée à une tonalité émotionnelle dans une interaction vivante.

${ }^{128}$ Selon Ph. Breton (2006), si l'interactivité est si souvent mise en avant, c'est parce que son attractivité est à la mesure de la frustration que les nouvelles formes de communication et de management ont suscitée.
} 
parfaitement intégrées dans le discours des personnes interviewées, elles demeurent essentiellement formulées en termes d'attentes qui «peinent à s'incarner dans un fonctionnement institutionnel complexe ». Cet écart entre le champ déclaratif et les pratiques évoquées par les cadres, témoigne de l'importance des questions de signification collective dans des institutions telles que les CAF. En termes d'évocations, plus de la moitié des personnes interrogées établissent un lien entre le concept d'institution communicante et «l'audace » qu'ils définissent comme la capacité de l'institution à participer au débat démocratique et social. Il faut d'ailleurs relever que 1' «audace» n'est pas ici mentionnée comme une valeur en contradiction avec la neutralité et l'objectivité, souvent invoquées dans les enquêtes pour qualifier l'institution (SOFRES/ Cnaf 2004; enquête salariés Cnaf, 2005), mais comme « un changement de culture à assumer » qui doit s'asseoir sur une vision claire des missions et un positionnement affirmé sur la scène publique. Les représentations des cadres traduisent ainsi une double attente au regard des valeurs portées par une institution qu'ils qualifieraient de «communicante». Celle-ci doit, selon eux, répondre aux nouvelles attentes sociales et communicationnelles des usagers et des personnels et elle doit le faire dans le respect des valeurs et de l'identité des services publics. Cette attente est soulignée par l'attachement des cadres à une approche qu'ils qualifient «d'éthique » de la communication.

Freins et plus-values liés, selon les cadres, à l'émergence de l'institution communicante

Les cadres des CAF voient dans l'hypothèse d'une institution où la communication jouerait un rôle facilitateur, la possibilité d'un fonctionnement en meilleure adéquation avec les valeurs de la société actuelle: «Nos usagers auraient le sentiment d'un fonctionnement plus clair, moins bureaucratique, qui reposerait sur des valeurs plus humaines, plus éthiques ». Ils se représentent également cette option comme un soutien à la performance: "Ça nous permettrait un fonctionnement plus serein et plus efficace ». Dans cette quête d'une communication en meilleure adéquation avec les attentes internes et externes, les cadres identifient des freins, notamment le fonctionnement "compliqué» de l'organisation des CAF : "Il faut clarifier les liens qui existent entre les CAF et l'Etat, mais aussi entre la Cnaf et le réseau des CAF». Enfin, la réorganisation en cours du réseau des CAF vers la mutualisation de certaines fonctions, dont la communication, représente, selon eux, un risque « de formatage et de recentralisation de la communication des $C A F »$ que certains 
considèrent comme une entrave pour une « communication adaptée, efficace et porteuse de sens ».

\section{Une tentative de modélisation de la notion d'institution communicante}

Comme nous venons de le voir, les résultats obtenus conduisent globalement à une certaine prudence quant à la validité de la notion d'institution communicante à propos des pratiques communicationnelles des CAF. Cependant, l'analyse des représentations des cadres a permis de dessiner les contours de cette même notion afin d'envisager sa transférabilité à d'autres organisations publiques. Cet essai de modélisation, que nous présentons ici (Fig.1), confirme l'interdépendance entre les dimensions organisationnelles, communicationnelles et managériales et montre que l'image des organisations publiques se construit dans une pluralité et une complexité d'interactions avec le contexte sociétal et institutionnel. Il montre par ailleurs que c'est la mise en perspective dialogique de trois approches qui fournit tout son intérêt à la notion d'institution communicante.

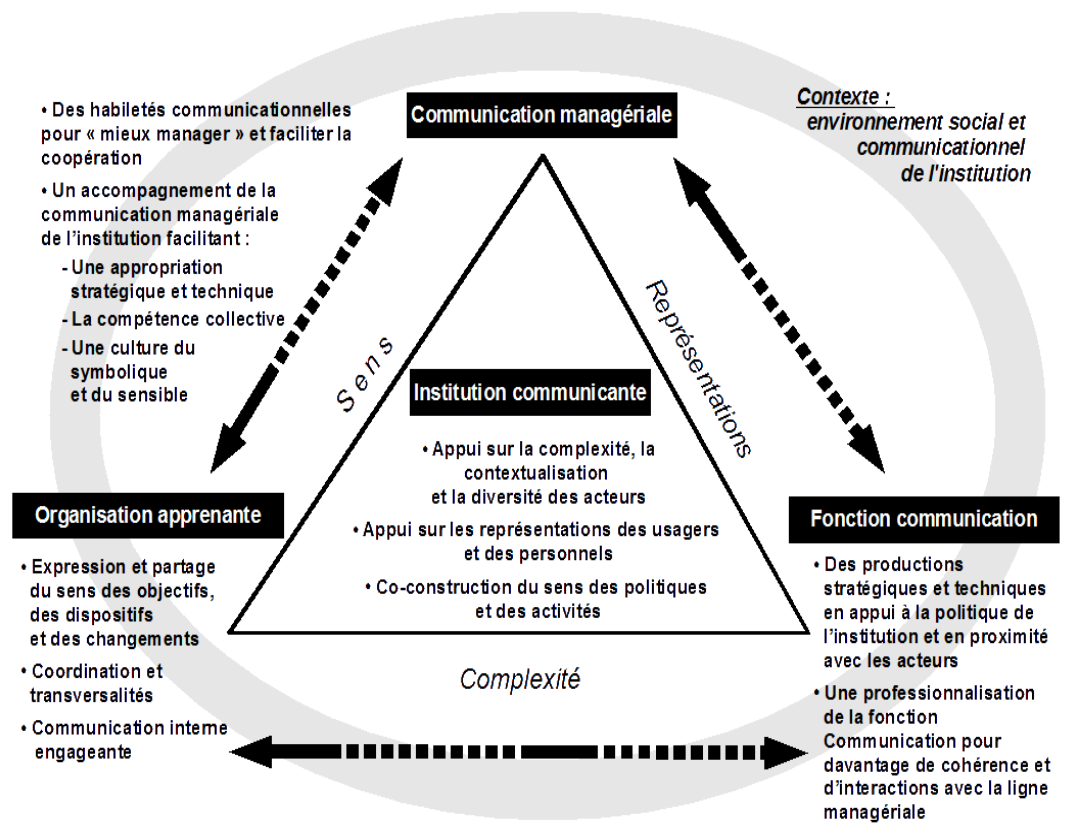

Fig.1 - Un modèle d'institution communicante élaboré à partir des représentations des Cadres des Caf 
La première se pose en termes de complexité fondée sur la compréhension des interactions fonctionnelles et symboliques orientées ou non vers la coopération, de prise en compte de la combinatoire culturelle et sociale au sein de l'organisme et de capacité de ce dernier à faciliter les formes d'innovation. La seconde approche engage la compréhension des représentations des acteurs de la communication interne et externe de l'organisme. Enfin, la troisième se situe au plan de la construction concertée et de l'appropriation des significations liées aux métiers, aux services et aux résultats visés, aux démarches et aux procédures retenues, auprès de l'ensemble des acteurs.

\section{La mise en perspective communicationnelle de l'organisation et du management des CAF par les cadres}

$\mathrm{Au}$ cours des entretiens, les cadres des CAF ont également précisé ce qu'ils attendent d'une institution communicante en ce qui concerne la communication managériale, l'organisation des activités de production et l'accompagnement de la fonction communication.

\section{Au plan de la communication managériale}

Si communiquer est perçu par la majorité des cadres comme un acte "plus complexe qu'il y a dix ans », leurs représentations ont confirmé que la communication managériale est encore peu pensée, dans les organisations publiques, en tant que levier pour favoriser l'intercompréhension et la transversalité. Dans ce contexte, selon les cadres, une logique déterministe et fonctionnelle prime encore beaucoup : si les orientations stratégiques des CAF sont prescrites et diffusées aux personnels, le sens de leurs finalités pour le service aux usagers ou l'organisation interne des organismes l'est beaucoup moins, du fait "d'une insuffisance de débat et de consultation». Le regard des cadres sur cette "carence en communication interne » autour des politiques d'organismes est à rapprocher d'une conception essentiellement innéiste et techniciste des compétences en communication managériale qui prévaut, selon eux, au sein des CAF.

Sur le plan de la communication managériale, un des éléments caractéristiques, selon les cadres, d'une institution communicante, concerne l'identification des "compétences qui permettent de mieux manager ", notamment pour répondre à des besoins de coopération et de co-responsabilité dans les relations managériales. Cela impliquerait, selon eux, d'envisager ces relations en termes de transversalité et d'échange à propos de la prescription institutionnelle de travail. 
Les organisations publiques...

\section{Au plan de l'organisation}

La forte interdépendance entre les questions de communication, de management et d'organisation a contraint les institutions à penser globalement la question du sens des projets, de l'implication des acteurs, des échanges et de la coopération dans les relations professionnelles. Pour autant, les institutions de « service public» sont encore très imprégnées du modèle d'organisation hiérarchique reposant sur la légitimité du commandement et induisant des relations et un système d'information très vertical. Dans le même temps, pour ces institutions, comme pour les entreprises du secteur privé, ce sont les organisations en réseau ${ }^{\mathbf{1 2 9}}$ qui sont aujourd'hui très valorisées dans un contexte de recherche d'adaptabilité et de gains de productivité. Au travers des représentations des cadres des CAF, il apparaît que pour se construire en tant qu'institutions communicantes, les organismes doivent davantage rechercher un modèle d'organisation apprenante au lieu de survaloriser un modèle d'organisation «en réseau ». Fondée sur «le modèle de la compétence » (Zarifian, 2001), l'organisation apprenante vise à construire, faire approprier et partager une même visée d'action et d'objectifs et à développer une communication « engageante ${ }^{130}$ ». Ainsi, il apparaît nécessaire aux cadres interrogés de rendre plus intelligible, pour les personnels et les publics, le sens des dispositifs et des changements mis en place. Sur ce plan, un modèle d'institution communicante s'attacherait donc "à donner $d u$ sens à l'organisation ».

\section{Au plan de la fonction communication}

Du point de vue des productions de la fonction communication, les cadres des CAF attendent que les schémas directeurs de communication (stratégies de communication des CAF) viennent davantage renforcer le projet global des organismes. Enfin, il faut souligner l'enjeu d'une proximité culturelle et fonctionnelle de la fonction communication avec les cadres de l'institution, notamment

\footnotetext{
${ }^{129}$ Dans un entretien accordé au Monde (2004), A. Lebaube constate que le concept de réseau s'est imposé durablement dans et autour de l'entreprise. Selon lui "L'organisation en réseau se caractérise par un ensemble d'entreprises qui fonctionnent comme une unité, sans perdre leur autonomie. Les échanges et les interactions s'y développent horizontalement et de manière décentralisée même s'il existe une hiérarchie voire un centre ».

130 Joulé et Beauvois (2004) distinguent quatre facteurs qui, en concourrant à la visibilité d'un acte, permettent de produire de l'engagement : caractère public, caractère explicite, irrévocabilité et répétition.
} 
au plan des valeurs, des pratiques professionnelles et du sentiment d'appartenance à l'organisme. Il s'agit là, selon les personnes interrogées, d'un facteur susceptible de renforcer la compétence collective des acteurs du management ainsi que la légitimité des professionnels de la communication.

\section{L'instauration du débat comme creuset d'une institution en quête de coopération et d'intercompréhension}

En proposant de convoquer la pensée de complexité pour appréhender la communication des organisations publiques, la notion d'institution communicante permet de donner à voir les liens d'interdépendance entre les questions d'organisation, de communication managériale et d'image dans un contexte qui impose une redéfinition de leur identité sur la scène sociale, en adéquation avec les nouvelles attentes des usagers et des personnels. Le modèle d'institution communicante permet également de penser assez finement les liens entre communication, organisation et management. En ce sens, il ouvre des pistes de problématisation en matière de coopération et d'intercompréhension entre la ligne managériale, les services et les personnels des organismes. A ce titre, il vient également questionner les effets sensibles et symboliques des pratiques managériales sur le terrain de l'identité professionnelle des acteurs, du sentiment d'appartenance à l'institution et de la qualité des relations interpersonnelles dans le travail. Enfin, cette notion permet de pointer la spécificité et la plus-value potentielle de la fonction communication des organismes. Parce qu'elles sont chargées d'incarner et de mettre en œuvre le projet social et démocratique, les institutions de service public sont porteuses de valeurs et d'attentes spécifiques de la part des usagers. Leur communication est inévitablement mise en tension par les débats sociaux et par ces attentes au regard de la sphère publique. Dans ce contexte, l'intérêt de la notion d'institution communicante est d'interroger la capacité de mise en dialogue des différentes dimensions de la communication des organismes envers les usagers, mais aussi de prendre en considération les représentations des personnels en termes d'appartenance, d'intercompréhension et de coopération. Enfin et plus globalement, il apparaît ici que l'attention portée aux représentations des usagers, des personnels et des partenaires, est de nature à (ré)installer de façon actualisée une problématique (re)fondatrice du paradigme communicationnel au sein des organisations publiques : celle du débat ouvert à une altérité dont le sens ne peut échapper à des acteurs évoluant dans le champ du social. 
Les organisations publiques...

\section{Bibliographie}

ABRIC J.-C., (sous la dir.), Méthodes d'études des représentations sociales, Erès, 2003.

BONEU F., L'entreprise communicante - Démarches et méthodes de communication interne, Paris, Les éditions Liaisons, 1990.

BONNET R., BONNET J., Nouvelles logiques, nouvelles compétences des cadres et des dirigeants - Entre le relationnel et le sensible, Paris, L'Harmattan, 2003.

BONNET R., BONNET J., Du manager novice au manager expert: quête de sens et parcours de professionnalisation, Paris, Hermès, 2006.

BRETON P., L'incompétence démocratique, la crise de la parole aux sources du malaise (dans la) politique, Paris, La découverte, 2006.

FISCHER G.-N., Les concepts fondamentaux de la psychologie sociale, Paris, Dunod, 2005.

D'IRIBARNE P., L'étrangeté française, Paris, Le Seuil, 2006.

LAMIZET B., Sémiotique de l'événement, Paris, Hermès, 2006

LIPOVETSKY G., La société de déception, Paris, Textuel, 2006.

MORIN E., Relier les connaissances, Paris, Seuil, 2000.

ORGOGOZO I., L'entreprise communicante, Des châteaux forts aux cloisons mobiles, Paris, Editions d'organisation, 1999.

VARELA F., Quels savoirs pour l'éthique?, Paris, La Découverte, 2004.

VERMERSCH P., L'entretien d'explicitation, Paris, ESF, 1994.

WOLTON D., Penser la communication, Paris, Flammarion, 2007

ZARIFIAN P., Travail et communication, Paris, PUF, 2001. 